Groups Geom. Dyn. 5 (2011), 443-450

DOI $10.4171 / \mathrm{GGD} / 189$
Groups, Geometry, and Dynamics

(C) European Mathematical Society

\title{
Connectivity of complexes of separating curves
}

\author{
Eduard Looijenga \\ In memory of Fritz Grunewald
}

\begin{abstract}
We prove that the separating curve complex of a closed orientable surface of genus $g$ is $(g-3)$-connected. We also obtain a connectivity property for a separating curve complex of the open surface that is obtained by removing a finite set from a closed one, where it is assumed that the removed set is endowed with a partition and that the separating curves respect that partition. These connectivity statements have implications for the algebraic topology of the moduli space of curves.
\end{abstract}

Mathematics Subject Classification (2010). 57N05, 55U15.

Keywords. Separating curve complex.

\section{Statements of the results}

Let $S$ be a connected oriented surface of genus $g$ with finite first Betti number $2 g+n$ (i.e., a closed surface with $n$ punctures) and make the customary assumption that $S$ has negative Euler characteristic: if $g=0$, then $n \geq 3$ and if $g=1$, then $n \geq 1$. We recall that the curve complex $\mathcal{C}(S)$ of $S$ is the simplicial complex whose vertex set consists of the isotopy classes of embedded (unoriented) circles in $S$ which do not bound in $S$ a disk or a cylinder. A finite set of vertices spans a simplex precisely when its elements can be represented by embedded circles that are pairwise disjoint. Thus, a closed 1-dimensional submanifold $A$ of $S$ with $k+1$ connected components such that every connected component of its complement has negative Euler characteristic defines a $k$-simplex $\sigma_{A}$ of $\ell(S)$ and every simplex of $\zeta(S)$ is thus obtained.

This complex has proven to be quite useful in the study of the mapping class group of $S$. For the purposes of studying the Torelli group of $S$ a subcomplex $\ell_{\text {sep }}(S)$ of $\digamma(S)$ can render a similar service. It is defined as the full subcomplex of $\mathcal{C}(S)$ spanned by the separating vertices of $\mathcal{C}(S)$, where a vertex is called separating if a representative embedded circle separates $S$ into two components. Our main result for the case when $S$ is closed is contained in the following theorem. 
Theorem 1.1 $\left(A_{g}\right)$. If $n \leq 1$, then the simplicial complex $\mathcal{C}_{\text {sep }}(S)$ is $(g-3)$ connected.

Previous work on this topic that we are aware of concerns the case $n=0$. Farb and Ivanov announced in 2005 [1], Theorem 4, that $\ell_{\text {sep }}(S)$ is connected for $g \geq 3$. Putman gave in [6], Theorem 1.4, another proof of this and showed that $\mathcal{C}_{\text {sep }}(S)$ is simply connected for $g \geq 4$ (op. cit., Theorem 1.11). In that paper he also mentions that Hatcher and Vogtmann have proved that $\mathcal{C}_{\text {sep }}(S)$ is $\left\lfloor\frac{1}{2}(g-3)\right\rfloor$-connected for all $g$ (unpublished).

Remark 1.2. Possibly the connectivity bound in Theorem 1.1 is the best possible for every positive genus. In a paper with Van der Kallen [3] we showed that the quotient of $\mathcal{C}_{\text {sep }}(S)$ by the action of the Torelli group of $S$ has the homotopy type of a bouquet of $(g-2)$-spheres. In the situation of the theorem, $\ell_{\text {sep }}(S)$ has dimension $2 g-4+n$ so that its connectivity is half the dimension (as $n \leq 1$ ). In particular, we cannot conclude that $\ell_{\text {sep }}(S)$ is spherical.

Before we state a version for the case $n \geq 2$, we point out a consequence that pertains to the moduli space of curves. Consider the Teichmüller space $\mathcal{T}(S)$ of $S$, a contractible manifold on which acts the mapping class group $\Gamma(S)$. The action is proper and the orbit space may be identified with the moduli space $\mathcal{M}_{g}$ of curves of genus $g$. The Harvey bordification of $\mathcal{T}(S)$, here denoted by $\mathcal{T}(S)^{+} \supset \mathcal{T}(S)$, is a (noncompact) manifold with boundary with corners to which the action of $\Gamma(S)$ naturally extends. This action is also proper and according to [4] the orbit space $\mathcal{M}_{g}^{+}:=\Gamma(S) \backslash \mathcal{T}(S)^{+}$is a compactification of $\mathcal{M}_{g}$ that can also be obtained from the Deligne-Mumford compactification $\widetilde{\mathcal{M}}_{g} \supset \mathcal{M}_{g}$ as a 'real oriented blowup' of its boundary $\Delta_{g}:=\widetilde{\mathcal{M}}_{g}-\mathcal{M}_{g}$. The walls of $\mathcal{T}(S)^{+}$define a closed covering of the boundary $\partial \mathcal{T}(S)^{+}$and any nonempty corner closure is an intersection of walls. As is well-known, the curve complex $\mathcal{C}(S)$ can be identified with the nerve of this covering of $\partial \mathcal{T}(S)^{+}$. Since the corner closures are contractible, Weil's nerve theorem implies that $\partial \mathcal{T}(S)^{+}$has the same homotopy type as $\mathcal{C}(S)$.

Let $\Delta_{g, 0} \subset \Delta_{g}$ denote the irreducible component of the Deligne-Mumford boundary whose generic point parameterizes irreducible curves with one singular point. We may understand $\mathcal{M}_{g}^{c}:=\widetilde{\mathcal{M}}_{g}-\Delta_{g, 0}$ as the moduli space of stable genus $g$ curves all of whose nodes are separating (which is equivalent to the irreducible components of the curve being smooth and with their genera summing up to $g$ ) and $\Delta_{g}^{c}:=\Delta_{g}-\Delta_{g, 0}$ as the locus in $\mathcal{M}_{g}^{c}$ that parameterizes the singular ones among them. If $\Gamma$ is a subgroup of $\Gamma(S)$ with the property that every Dehn twist along a separating curve in $S$ has a positive power lying in $\Gamma$, then this defines a (not necessarily finite) cover $\widetilde{\mathcal{M}}_{g}^{c} \rightarrow \mathcal{M}_{g}^{c}$.

Corollary 1.3. Suppose that $\Gamma \subset \Gamma(S)$ is as above and is in addition torsion-free. If we denote by $\widetilde{\Delta}_{g}^{c} \subset \widetilde{\mathcal{M}}_{g}^{c}$ the preimage of $\Delta_{g}^{c}$, then the pair $\left(\widetilde{\mathcal{M}}_{g}^{c}, \widetilde{\Delta}_{g}^{c}\right)$ is $(g-2)$ connected. Moreover, $H_{k}\left(\mathcal{M}_{g}^{c}, \Delta_{g}^{c} ; \mathbb{Q}\right)=0$ for $k \leq g-2$. 
Proof. Let $\mathcal{T}(S)_{\text {sep }}^{+}$be obtained from $\mathcal{T}(S)^{+}$by removing the walls that correspond to the nonseparating vertices of $\mathcal{C}(S)$. Then $\mathcal{T}(S)_{\text {sep }}^{+}$is the preimage of $\mathcal{M}_{g}^{c}$ in $\mathcal{T}(S)^{+}$. The same reasoning as above shows that $\partial \mathcal{T}(S)_{\text {sep }}^{+}$is homotopy equivalent to $\mathscr{C}(S)_{\text {sep }}$ and so $\partial \mathcal{T}(S)_{\text {sep }}^{+}$is $(g-3)$-connected. It follows that we can construct a relative $\mathrm{CW}$ complex $\left(Z, \partial \mathcal{T}(S)_{\text {sep }}^{+}\right)$obtained from $\partial \mathcal{T}(S)_{\text {sep }}^{+}$by attaching cells of dimension $\geq g-1$ in a $\Gamma(S)$-equivariant manner as to ensure that $Z$ is contractible and no nontrivial element of $\Gamma(S)$ fixes a cell. Then $\Gamma$ acts freely on $Z$ (as it does on the contractible space $\left.\mathcal{T}(S)_{\text {sep }}^{+}\right)$and so there is a $\Gamma$-equivariant homotopy equivalence $Z \rightarrow \mathcal{T}(S)_{\text {sep }}^{+}$relative to $\partial \mathcal{T}(S)_{\text {sep }}^{+}$. It follows that we also have a homotopy equivalence $\Gamma \backslash Z \rightarrow \widetilde{\mathcal{M}}_{g}^{c}$ relative to $\widetilde{\Delta}_{g}^{c}$ and we conclude that $\left(\widetilde{\mathcal{M}}_{g}^{c}, \widetilde{\Delta}_{g}^{c}\right)$ is $(g-2)$-connected.

The last assertion follows from the existence of a normal subgroup $\Gamma \subset \Gamma(S)$ of finite index that is torsion-free. For if $\Gamma$ is such a group, then $H_{k}\left(\mathcal{M}_{g}^{c}, \Delta_{g}^{c} ; \mathbb{Q}\right) \cong$ $H_{k}\left(\tilde{\mathcal{M}}_{g}^{c}, \widetilde{\Delta}_{g}^{c} ; \mathbb{Q}\right)^{\Gamma(S) / \Gamma}=0$ for $k \leq g-2$.

A similar statement holds for the universal curve $\mathcal{M}_{g, 1}$.

When $n>1$, we need to come to terms with the fact that the separability notion has no good hereditary properties: if $T$ is a closed surface, $A \subset T$ a compact 1-dimensional submanifold representing a simplex of $\mathcal{C}(T)$ and $S$ a connected component of $T-A$, then a vertex of $\mathcal{C}(S)$ may split $S$, but not $T$. This happens precisely when the vertex in question separates two boundary components of $\partial S$ that lie on the same connected component of $T-S$. So the basic object should be, what Andy Putman calls in [5], a partitioned surface: a closed surface minus a finite set, for which the removed set comes with a partition. This leads to the following definition.

Definition 1.4. Let $N$ be the set of points of $S$ at infinity (the cusps) and let $P$ be a partition of $N$. We call a vertex of $\mathcal{C}(S)$ separating relative to $P$ if a representative embedded circle $\alpha \subset S$ has the property that $S-\alpha$ has two connected components each of which meets $N$ in a union of parts of $P$. We denote by $\mathcal{C}(S, P)$ the full subcomplex of $\mathcal{C}(S)$ spanned by such vertices.

One might also understand $\mathcal{C}(S, P)$ as the full subcomplex of $\mathcal{C}(S)$ spanned by the isotopy classes of embedded cycles which are separating on the surface $S^{P}$ that is obtained by capping off for each part of $P$ the corresponding set of cusps by a sphere with that many holes. Notice that $\mathcal{C}(S, P) \subset \mathcal{C}_{\text {sep }}(S)$ and that we have equality when $P$ is discrete or $N$ is empty.

We shall prove Theorem 1.1 by induction and simultaneously with

Theorem $1.5\left(A_{g, n}\right)$. Suppose that $g>0$ and $n=|N|>1$. Let $P$ be a partition of $N$. Then $\mathcal{C}(S, P)$ is $(g-2)$-connected.

To be precise, the induction starts with $g=0$, where the statements $\left(A_{g}\right)$ and $\left(A_{0, n}\right)$ are trivially true and the induction strategy will be to show that 
(i) $\left(A_{h, n}\right)$ for $h<g$ implies $\left(A_{g}\right)$, and

(ii) $\left(A_{g}\right)$ and $\left(A_{h, k}\right)$ for $(h, k)<(g, n)$ (for the lexicographic ordering) imply $\left(A_{g, n}\right)$.

I am indebted to Allen Hatcher for pointing out that the stronger version of Theorem 1.5 that I stated in a previous version was incorrect. Yet it may be that some such statement might hold. For instance, if $r(P)$ denotes the number of nonempty parts of $P$ and $s(P)$ the number of parts with at least two elements, is it true that $\mathcal{C}(S, P)$ is $(g+r(P)+s(P)-4)$-connected when $g>0$ (as I claimed in the earlier version)? In case $g=0, \mathcal{C}(S, P)$ is a complex of dimension $r(P)+s(P)-4$. Is this $(r(P)+s(P)-5)$-connected? In other words, is this complex spherical?

I am grateful to the referee, whose meticulous job helped to improve the paper. The proof of Lemma 2.3 follows a suggestion by the referee and simplifies my original one.

I also gratefully acknowledge support by the Mathematical Sciences Center of Tsinghua University at Beijing, where some of this work was done.

\section{Proofs}

Before we start off, we mention the following elementary fact that we will frequently use.

Lemma 2.1. Let $X_{i}$ be a $d_{i}$-connected space $\left(d_{i}=-1\right.$ means $\left.X_{i} \neq \emptyset\right)$, where $i=1, \ldots, k$. Then the iterated join $X_{1} * \cdots * X_{k}$ is $\left(-2+\sum_{i=1}^{k}\left(d_{i}+2\right)\right)$-connected.

Proof that $\left(A_{h, n}\right)$ for $h<g$, all $n$, implies $\left(A_{g}\right)$. So here $n \leq 1$. We must show that $\mathcal{C}_{\text {sep }}(S)$ is $(g-3)$-connected. For $g<2$, there is nothing to show and so we may assume that $g \geq 2$. A theorem of Harer [2, Thm. 1.2] asserts that $\mathcal{C}(S)$ is $(2 g-3)$ connected. So it is certainly $(g-3)$-connected. Let $\mathcal{C}_{k}$ be the subcomplex of $\mathcal{C}(S)$ that is the union of $\mathcal{C}_{\text {sep }}(S)$ and the $k$-skeleton of $\mathscr{C}(S)$. So $\mathcal{C}_{-1}=\mathscr{C}_{\text {sep }}(S)$ and $\ell_{k}=\mathscr{C}(S)$ for $k$ large. Notice that a finite set of vertices of $\mathcal{C}(S)$ spans a simplex of $\ell_{k}$ if and only if no more than $k+1$ of these are nonseparating. Hence a minimal simplex of $\ell_{k}-\ell_{k-1}$ is represented by a compact 1-dimensional submanifold $A \subset S$ with $k+1$ connected components, each of which is nonseparating. We prove that the boundary of the star of such a simplex in $\ell_{k}$ is a $(g-3)$-connected subcomplex of $\mathcal{C}_{k-1}$. This property implies that the pair $\left(\mathcal{C}(S), \mathcal{C}_{\text {sep }}(S)\right)$ is $(g-2)$-connected. Since $\mathcal{C}(S)$ is $(g-3)$-connected, it then follows that $\mathcal{C}_{\text {sep }}(S)$ is. Let $\left\{S_{i}\right\}_{i \in I}$ be the set of connected components of $S-A$. Notice that if $g_{i}$ is the genus of $S_{i}$, then $g_{i}<g$. An Euler characteristic argument shows that

$$
g-1=k+1+\sum_{i \in I}\left(g_{i}-1\right) .
$$


We denote by $N_{i}$ the set of 'cusps' of $S_{i}$, i.e., the finitely many points needed to make $S_{i}$ a closed surface. So an element of $N_{i}$ is given by possibly a cusp of $S$ (if it exists and if it is also a cusp of $S_{i}$ ) or by a connected component of $A$ in the boundary of $S_{i}$ endowed with the orientation it receives as such. The set $N_{i}$ comes with an evident partition $P_{i}$ : if $S$ has a cusp and $N_{i}$ contains it, then this cusp makes up a singleton part of $P_{i}$ and any other two elements of $N_{i}$ belong to the same part of $P_{i}$ if and only if they come from connected components of $A$ that lie on the same connected component of $S-S_{i}$. (NB: beware that a connected component of $S-S_{i}$ could be simply a connected component $A_{o}$ of $A$; then its two orientations define a 2-element part of $P_{i}$.) connected components of $A$ that bound $S_{i}$. Note that since the connected components of $A$ are nonseparating, we always have $\left|N_{i}\right| \geq 2$. By our induction hypothesis $\mathcal{C}\left(S_{i}, P_{i}\right)$ is then $\left(g_{i}-2\right)$-connected. The boundary of the star of the $k$-simplex $\sigma_{A}$ defined by $A$ in $\ell_{k}$ lies in $\ell_{k-1}$ and can be identified with the $(|I|+1)$-fold join

$$
\partial \sigma_{A} *\left(\underset{i \in I}{*} \varphi\left(S_{i}, P_{i}\right)\right)
$$

Since $\partial \sigma_{A}$ is a combinatorial $(k-1)$-sphere, this join has by Lemma 2.1 connectivity at least $-2+k+\sum_{i \in I} g_{i}$. By the displayed formula above this is equal to $g-4+|I|$ and is therefore $\geq g-3$.

The proof of $A_{g, n}$ begins with a discussion. We now assume that $g>0$ and $n \geq 2$. Let $x \in N$. Notice that $S^{\prime}:=S \cup\{x\}$ has still negative Euler characteristic. We put $N^{\prime}:=N-\{x\}$ and $P^{\prime}:=P \mid N^{\prime}$. The goal is to compare $\ell\left(S^{\prime}, P^{\prime}\right)$ with $\digamma(S, P)$. There is in general no forgetful map $\ell(S, P) \rightarrow \digamma\left(S^{\prime}, P^{\prime}\right)$ because there will be vertices of $\mathcal{C}(S, P)$ that do not give vertices of $\mathcal{C}\left(S^{\prime}, P^{\prime}\right)$. Let us first identify this set of vertices.

Denote by $\Sigma_{x} \subset N-\{x\}$ the set of $y \in N-\{x\}$ for which $\{x, y\}$ is a union of parts of $P$. In other words, if $P_{x}$ denotes the part of $P$ that contains $x$, then $\Sigma_{x}$ is empty if $P_{x}$ has more than 2 elements, equals $P_{x}-\{x\}$ if $P_{x}$ is a 2-element set, and equals the set of $y \neq x$ for which $P_{y}$ is a singleton in case $P_{x}=\{x\}$. Then the vertices of $\mathcal{C}(S, P)$ that have no image in $\ell\left(S^{\prime}, P^{\prime}\right)$ are precisely the vertices $\alpha$ of $\ell_{\text {sep }}(S)$ which for some $y \in \Sigma_{x}$ bound a disk neighborhood of $\{x, y\}$ in $S \cup\{x, y\}$. Such a disk neighborhood can be thought of as a regular neighborhood of an arc in $S \cup\{x, y\}$ connecting the two added cusps; this may help to explain why we have chosen to denote this set of vertices by $\operatorname{arc}_{(S, P)}(x)$. Denote by $\ell(S, P)_{x}$ the full subcomplex of $\mathcal{C}(S, P)$ spanned by the vertices not in $\operatorname{arc}_{(S, P)}(x)$.

Observe that $\operatorname{arc}_{(S, P)}(x)$ is empty (so that $\ell(S, P)_{x}=\ell(S, P)$ ) if $\Sigma_{x}$ is.

Lemma 2.2. The link in $\mathcal{C}(S, P)$ of every vertex of $\operatorname{arc}_{(S, P)}(x)$ is a subcomplex of $\ell(S, P)_{x}$ that projects isomorphically onto $e\left(S^{\prime}, P^{\prime}\right)$.

Proof. A vertex of $\operatorname{arc}_{(S, P)}(x)$ defines a $y \in \Sigma_{x}$ and (up to isotopy) a closed disk $D$ in $S \cup\{x, y\}$ that is a neighborhood of $\{x, y\}$. The inclusion $S-D \subset S^{\prime}$ identifies the link in question with $\mathcal{C}\left(S^{\prime}, P^{\prime}\right)$. 
Denote by $\widetilde{P}$ the refinement of $P$ which coincides with $P$ on $N-P_{x}$ and partitions $P_{x}$ further into $\{x\}$ and $P_{x}-\{x\}$. So $\widetilde{P}^{\prime}=P^{\prime}$. It is clear that $\mathcal{C}(S, P)$ is a subcomplex of $\mathcal{C}(S, \widetilde{P})$. Notice that $\operatorname{arc}_{(S, P)}(x)=\mathcal{C}(S, P) \cap \operatorname{arc}_{(S, \widetilde{P})}(x)$ (we have $\operatorname{arc}_{(S, P)}(x)=\operatorname{arc}_{(S, \widetilde{P})}(x)$ unless $\left.\left|P_{x}\right|=2\right)$ and $\mathcal{C}(S, P)_{x}=\mathcal{C}(S, P) \cap \mathcal{C}(S, \widetilde{P})_{x}$. We denote by $f$ the forgetful simplicial map $\mathcal{C}(S, \widetilde{P})_{x} \rightarrow \mathcal{C}\left(S^{\prime}, P^{\prime}\right)$ so that we have the diagram

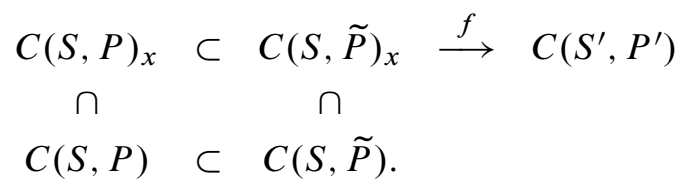

Lemma 2.3. The map $f: \mathcal{C}(S, \widetilde{P})_{x} \rightarrow \mathcal{C}\left(S^{\prime}, P^{\prime}\right)$ is a homotopy equivalence.

Proof. Choose an arc $\gamma$ which connects $x$ with another point of $N$ and defines a vertex of $\operatorname{arc}_{(S, P)}(x)$ and observe that the full subcomplex $K \subset \mathcal{C}(S, \widetilde{P})_{x}$ spanned by vertices that avoid $\gamma$ defines a section of $f$ (the inclusion $S-\gamma \subset S^{\prime}$ is isotopic to a homeomorphism). We shall prove that $\mathcal{C}(S, \widetilde{P})_{x}$ admits $K$ as a deformation retract. (The proof will in fact show that each fiber of $|f|$ is a tree and essentially produces for every element of $\left|\mathcal{C}(S, \widetilde{P})_{x}\right|$ the unique path in its $|f|$-fibre that connects it to the point of $|K|$.)

Denote by $K_{r} \subset \mathcal{E}(S, \widetilde{P})_{x}$ the subcomplex whose simplices can be represented by a closed submanifold $A \subset S$ which meets $\gamma$ transversally in at most $r$ points. This defines a filtration $K=K_{0} \subset K_{1} \subset K_{2} \subset \cdots$ whose union is $\mathcal{C}(S, \widetilde{P})_{x}$. Although this filtration is infinite, it is enough to construct for every $r \geq 0$ a deformation retraction of $\left|K_{r+1}\right|$ onto $\left|K_{r}\right|$, for in the simplicial setting an infinite sequence of deformation retractions still gives a deformation retraction.

We do this per simplex: if $\sigma$ is a simplex of $K_{r+1}$ that is not in $K_{r}$ and is minimal for this property, then its link in $K_{r+1}$ lies in $K_{r}$ and so it suffices to define for such a $\sigma$ a deformation retraction $h_{\sigma}$ of $\left|\operatorname{Star}_{K_{r+1}}(\sigma)\right|$ onto $\left|\operatorname{Link}_{K_{r+1}}(\sigma)\right|$.

The simplex $\sigma$ is represented by a closed submanifold $A \subset S$ of which every connected component meets $\gamma$ transversally and is such that $A \cap \gamma$ has cardinality $r+1$ (a number that cannot be made smaller in its isotopy class). Let $x_{0}$ be the point of $A \cap \gamma$ closest to $x$. Denote by $\alpha_{0}$ the connected component of $A$ which contains $x_{0}$ and choose in $S^{\prime}$ a thin regular neighborhood of the union of $\alpha_{0}$ and the subarc of $\gamma$ which connects $x_{0}$ with $x$. The boundary of that neighborhood has two connected components. Both lie in $S$ and only one of them is isotopic to $\alpha_{0}$. Denote by $\alpha_{0}^{\prime}$ the other boundary component. If $\tau$ is a simplex of $K_{r+1}$ which contains $\sigma$, then adding $\alpha_{0}^{\prime}$ to $\tau$ gives also a simplex $\tau^{\prime}$ of $K_{r+1}$ and the codimension one face $\tau^{\prime \prime}$ of $\tau^{\prime}$ obtained by removing $\alpha_{0}$ is contained in $K_{r}$. So if we regard $\left|\operatorname{Star}_{K_{r+1}}(\sigma)\right|$ as the cone over $\left|\operatorname{Link}_{K_{r+1}}(\sigma)\right|$ with the barycenter of $\sigma$ as its vertex, then there is a simplicial map from this cone to its base which sends the barycenter to $\alpha_{0}^{\prime}$ and is the identity on the base. Its geometric realization yields the desired $h_{\sigma}$. 
Corollary 2.4. The complex $\mathcal{C}(S, P) \cup \mathcal{C}(S, \widetilde{P})_{x}$ is canonically homotopy equivalent to the join $\operatorname{arc}_{(S, P)}(x) * \mathcal{C}\left(S^{\prime}, P^{\prime}\right)\left(\right.$ where $\operatorname{arc}_{(S, P)}(x)$ is discrete $)$.

Proof. The set of vertices of $\mathcal{C}(S, P) \cup \mathcal{C}(S, \widetilde{P})_{x}$ not in $\ell(S, \widetilde{P})_{x}$ is $\operatorname{arc}_{(S, P)}(x)$. The link of any such vertex is contained in $\mathscr{C}(S, \widetilde{P})_{x}$ and by Lemma 2.2 that link projects isomorphically onto $e\left(S^{\prime}, P^{\prime}\right)$. In view of Lemma 2.3 this implies that the inclusion of this link in $\mathcal{C}(S, \widetilde{P})_{x}$ is also a homotopy equivalence. Hence the natural inclusion $\mathcal{C}(S, P) \cup \mathcal{C}(S, \widetilde{P})_{x} \subset \operatorname{arc}_{(S, P)}(x) * \mathcal{C}(S, \widetilde{P})_{x}$ is a homotopy equivalence. The corollary follows.

From now on we assume that $A_{g}$ holds and that $A_{h, k}$ holds for all $(h, k)$ smaller than $(g, n)$ for the lexicographic ordering. Our goal is to prove $A_{g, n}$.

Lemma 2.5. The pair $\left(\mathcal{C}(S, P) \cup \mathcal{C}(S, \widetilde{P})_{x}, \mathcal{C}(S, P)\right)$ is $(g-1)$-connected.

Proof. If $P_{x}=\{x\}$, then $\widetilde{P}=P$ and there is nothing to show. We therefore assume that $P_{x}$ has more than one element. Denote by $\ell_{k}$ the subcomplex of $\ell(S, P) \cup$ $\digamma(S, \widetilde{P})_{x}$ that is the union of $\mathcal{C}(S, P)$ and the $k$-skeleton of $\mathcal{C}(S, P) \cup \mathcal{C}(S, \widetilde{P})_{x}$ : a finite set of vertices of $\mathcal{C}(S, P) \cup \mathcal{C}(S, \widetilde{P})_{x}$ spans a simplex of $\mathcal{C}_{k}$ if and only if no more than $k+1$ of these separate $x$ from $P_{x}-\{x\}$. Notice that $\ell_{-1}=\mathcal{C}(S, P)$

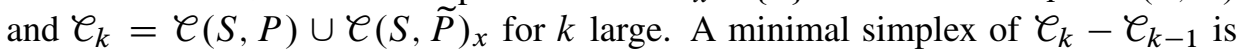
represented by a compact 1-dimensional submanifold $A \subset S$ with $k+1$ connected components, each of which separates $x$ from $P_{x}-\{x\}$ (the graph that is associated to $A$ is then a string with $k+2$ nodes). We prove that the boundary of the star of such a simplex in $\ell_{k-1}$ is $(g-2)$-connected if $g>0$. We enumerate the connected components of $A$ as $\alpha_{0}, \ldots, \alpha_{k}$ and the connected components of $S-A$ as $S_{0}, \ldots$, $S_{k+1}$ such that $\alpha_{i}$ is a boundary component of $S_{i}$ and $S_{i+1}$ and so that $S_{0}$ resp. $S_{k+1}$ is punctured by $x$ resp. $P_{x}-\{x\}$. The cusps of $S-A$ are naturally indexed by $\widehat{N}:=N \sqcup\left\{i_{ \pm}\right\}_{i=0}^{k}$, where $i_{-}$resp. $i_{+}$corresponds to the cusp defined by $\alpha_{i}$ on $S_{i}$ resp. $S_{i+1}$. Let $\widehat{N}_{i} \subset \widehat{N}$ index the set of cusps on $S_{i}$. Denote by $P_{i}$ the partition of $\left(N-P_{x}\right) \cap S_{i}$ that is simply the restriction of $P$ and denote by $\hat{P}_{i}$ the partition of $\hat{N}_{i}$ that on $\left(N-P_{x}\right) \cap S_{i}$ is equal to $P_{i}$ and has what remains of $\hat{N}_{i}$ as a single part. So this new part is $\left\{x, 0_{+}\right\}$for $i=0,\left\{(i-1)_{-}, i_{+}\right\}$for $0<i<k+1$ and $\left(P_{x}-\{x\}\right) \cup\left\{k_{-}\right\}$for $i=k+1$.

The reason for introducing these partitions is that we can now observe that the boundary of the star of the $k$-simplex $\sigma_{A}$ defined by $A$ in $\ell_{k}$ lies in $\ell_{k-1}$ and can be identified with the iterated join

$$
\partial \sigma_{A} * \ell\left(S_{0}, \hat{P}_{0}\right) * \cdots * \ell\left(S_{k+1}, \widehat{P}_{k+1}\right) .
$$

It is then enough to show that this join is $(g-2)$-connected for $g>0$. Since $\partial \sigma_{A}$ is a $(k-1)$-sphere, it is $(k-2)$-connected. The connectivity of a factor $C\left(S_{i}, \widehat{P}_{i}\right)$ with $g_{i}>0$ is at least $g_{i}-2$. So by Lemma 2.1 the connectivity of the above join is at least $-2+k+\sum_{\left\{i: g_{i}>0\right\}} g_{i}=g+k-2 \geq g-2$. 
Proof of $\left(A_{g, n}\right)$. We must show that $\mathcal{C}(S, P)$ is $(g-2)$-connected. In view of Lemma 2.5 it suffices to show that $\mathcal{C}(S, P) \cup \mathcal{C}(S, \widetilde{P})_{x}$ has that property.

If $\operatorname{arc}_{(S, P)}(x)=\emptyset$, then $n>2$ and so our induction hypothesis implies that $\mathcal{C}\left(S^{\prime}, P^{\prime}\right)$ is $(g-2)$-connected by $A_{g, n-1}$. It follows from Corollary 2.4 that $\mathcal{C}(S, P) \cup \mathcal{C}(S, \widetilde{P})_{x}$ is homotopy equivalent to $\mathcal{C}\left(S^{\prime}, P^{\prime}\right)$ and hence is $(g-2)$ connected.

If $\operatorname{arc}_{(S, P)}(x) \neq \emptyset$, then we may have $n=2$. At least we know that $\mathcal{C}\left(S^{\prime}, P^{\prime}\right)$ is $(g-3)$-connected (invoke $A_{g}$ if $\left.n=2\right)$. But since $\mathcal{C}(S, P) \cup \mathcal{C}(S, \widetilde{P})_{x}$ is homotopy equivalent to $\operatorname{arc}_{(S, P)}(x) * \mathcal{C}\left(S^{\prime}, P^{\prime}\right)$ (by Corollary 2.4), it is $(g-2)$-connected.

\section{References}

[1] B. Farb and N. V. Ivanov, The Torelli geometry and its applications: research announcement. Math. Res. Lett. 12 (2005), 293-301. Zbl 1073.57012 MR 2150885

[2] J. L. Harer, Stability of the homology of the mapping class groups of orientable surfaces. Ann. of Math. (2) 121 (1985), 215-249. Zbl 0579.57005 MR 786348

[3] W. van der Kallen and E. Looijenga, Spherical complexes attached to symplectic lattices. Geom. Dedicata 152 (2011), 197-211. Zbl 1217.05227 MR 2795243

[4] E. Looijenga, Cellular decompositions of compactified moduli spaces of pointed curves. In The moduli space of curves (Texel Island, 1994), Progr. Math. 129, Birkhäuser, Boston 1995, 369-400. Zbl 0862.14017 MR 1363063

[5] A. Putman, Cutting and pasting in the Torelli group. Geom. Topol. 11 (2007), 829-865. Zbl 1157.57010 MR 2302503

[6] A. Putman, A note on the connectivity of certain complexes associated to surfaces. Enseign. Math. (2) 54 (2008), 287-301. Zbl 1182.57004 MR 2478089

Received January 7, 2010; revised January 23, 2012

E. Looijenga, Mathematisch Instituut, Universiteit Utrecht, P.O. Box 80.010, 3508 TA Utrecht, The Netherlands

E-mail: E.J.N.Looijenga@uu.nl 